\title{
"Sem Pressão": aplicativo com orientações para identificação, estadiamento e prevenção de lesões por pressão
}

\section{"Sem Pressão": application with guidelines for identification, staging and prevention of pressure injuries}

\section{"Sem Pressão": aplicación con orientaciones para identificación, classificación y prevención de lesiones por presión}

Rosana Siqueira Campos ${ }^{1, *}$, Leila Blanes', Denise Nicodemo¹, Lydia Masako Ferreira

\section{ORCID IDS}

Campos RS (D) https://orcid.org/0000-0002-8905-3939

Blanes L (D) https://orcid.org/0000-0002-6922-7719

Nicodemo D (D) http://orcid.org/0000-0002-0510-009X

Ferreira LM (D) https://orcid.org/0000-0003-4587-509X

\section{COMO CITAR}

Campos RS; Blanes L; Nicodemo D; Ferreira LM. "Sem Pressão": aplicativo com orientações para identificação, estadiamento e prevenção de lesões por pressão. ESTIMA, Braz. J. Enterostomal Ther., 2020, 18: e3120. https://doi.org/10.30886/estima.v18.944_PT

\section{RESUMO}

Objetivo: desenvolver aplicativo com orientações sobre identificação, estadiamento e prevenção de lesões por pressão em adultos. Método: o método descritivo utilizado iniciou com a busca de aplicativos na área de lesões por pressão nas plataformas Google@ e Apple@. Após, foi desenvolvido o conteúdo, baseado, principalmente, nas recomendações da National Pressure Injury Advisory Panel. Foi criado um protótipo em www.fábricadeaplicativos.com.br e enviado para avaliação de forma e conteúdo. Seis profissionais de saúde preencheram um questionário avaliando o aplicativo e dois deles sugeriram alterações. O aplicativo foi adequado às sugestões e reavaliado por três enfermeiros estomaterapeutas que não sugeriram alterações. Resultados: $\circ$ aplicativo foi desenvolvido no site "fábrica de aplicativos" e denominado "Sem Pressão", com sete abas. Conclusão: foi criado o aplicativo com orientações sobre identificação, estadiamento e prevenção de lesões por pressão em adultos, e disponibilizado para download gratuito.

DESCRITORES: Lesão por pressão; Tecnologia da informação; Desenvolvimento tecnológico; Smartphone; Ferimentos e lesões.

\footnotetext{
1. Universidade Federal de São Paulo - Escola Paulista de Medicina - Programa de Pós-Graduação em Ciência, Tecnologia e Gestão Aplicadas à Regeneração Tecidual - São Paulo (SP), Brasil.
}

*Autora correspondente: ladynurse@gmail.com

Recebido: Set. 24, 2020 | Aceito: Nov. 06, 2020 
ABSTRACT

Objective: develop an application with guidance on the identification, staging and prevention of pressure injuries in adults. Method: the descriptive method used started with the search for applications in the area of pressure injuries on the Google (c) and Apple (c) platforms. Afterwards, the content was developed, based mainly on the recommendations of the National Pressure Injury Advisory Panel. A prototype was created at www.fábricadeaplicativos.com.br and sent for evaluation of form and content. Six health professionals completed a questionnaire evaluating the application and two of them suggested changes. The application was adequate to the suggestions and reassessed by three stomatherapist nurses who did not suggest changes. Results: the application was developed on the "application factory" website and named "Sem Pressão" (No Pressure), with seven tabs. Conclusion: the application was created with guidelines on identification, staging and prevention of pressure injuries in adults, and made available for free download.

DESCRIPTORS: Pressure injury; Information Technology; Technological development; Smartphone; Wounds and injuries.

\section{RESUMEN}

Introducción: Lesiones por Presión representan una de las principales complicaciones en pacientes hospitalizados, prolongando la internación y aumentando los costos globales de la asistencia. Recursos para orientación de profesionales, como sitios web y directrices apoyan en la identificación, prevención y classificación. La aplicación para teléfonos inteligentes se presenta como una estrategia educativa para detener estos profesionales y la ausencia de esta tecnología libre y en portugués instigado este trabajo. Objetivo: Desarrollar aplicación con orientaciones sobre identificación, classificación y prevención de Lesiones por Presión en adultos. Método: El método descriptivo utilizado inició con la búsqueda de aplicaciones en el área de Lesiones por Presión en las plataformas Google (c) y Apple ( ). Después, se desarrolló el contenido, baseado principalmente en las recomendaciones del National Pressure Injury Advisory Panel. Se creó un prototipo en www.fábricadeaplicativos.com.br y enviado para evaluación de forma y contenido. Seis profesionales de la salud llenaron un cuestionario evaluando la aplicación y dos de ellos sugirieron cambios. La aplicación fue adecuada a las sugerencias y reevaluado por 3 enfermeros estomaterapeutas, que no sugirieron cambios. Resultado: La aplicación fue desarrollada en el sitio "fabricadeaplicativos.com.br" y se denominó "Sem Pressão", con siete pestañas (http://app.vc/sem_pressao). Conclusión: Se creó la aplicación "Sem Pressão", con orientaciones sobre identificación, classificación y prevención de Lesiones por Presión en adultos y disponible para descarga gratuita.

DESCRIPTORES: Úlcera por Presión; Tecnología de la Información; Desarrollo tecnológico; Teléfono inteligente; Heridas y lesiones.

\section{INTRODUÇÃO}

Lesões por pressão (LP) são definidas como lesões localizadas na pele e/ou tecido subjacente, geralmente sobre proeminência óssea, resultantes da pressão isolada ou de pressão combinada com friç̧ão e/ou cisalhamento. Inúmeros fatores contribuintes podem estar associados às LP, mas o significado deles ainda deve ser elucidado ${ }^{1,2}$. Essas lesões podem apresentar-se como pele íntegra ou aberta e podem ser dolorosas. A tolerância do tecido à pressão pode ser influenciada pelo microclima, pela nutrição, perfusão tecidual, por comorbidades e pelas condições próprias do tecido ${ }^{1}$.

As LP também podem estar relacionadas ao uso de dispositivos médicos. São áreas localizadas na pele ou tecido subjacente como resultado da pressão sustentada de um dispositivo ${ }^{1}$. As lesões nos tecidos moles geralmente reproduzem a forma do dispositivo, que geralmente é rígido ou fixo com curativos apertados. Essas LP podem evoluir para lesões de espessura total devido à falta de tecido adiposo em muitas das áreas de ulceração ${ }^{3,4}$. Pacientes críticos são suscetíveis ao desenvolvimento de LP relacionadas a dispositivos por diversas razões, tais como edema, falta de sensibilidade e vasoconstrição periférica ${ }^{3}$.

Fatores de risco para desenvolvimento dessas lesões estão relacionados com a mobilidade, a umidade, a atividade, a percepção sensorial, o padrão nutricional, a fricção e o cisalhamento ${ }^{1}$. Os pacientes mais suscetíveis são os que se encontram em situação de imobilidade, tanto na cama como em cadeira de rodas, sejam idosos ou jovens acometidos de algum comprometimento sensorial, como traumatismos, coma, processos cirúrgicos extensos, entre outros ${ }^{5}$.

As LP são consideradas um importante problema de saúde da população em geral, pois representam uma das principais complicações que acometem pacientes hospitalizados, prolongando a internação e aumentando os custos globais da assistência ${ }^{6}$. Também são consideradas um indicador de qualidade da assistência nos serviços de saúde ${ }^{5,7}$ e classificadas internacionalmente como eventos adversos e representam desafio, pois aumentam a morbidade, 
a mortalidade, a duração e os custos do tratamento ${ }^{8}$, além de interferirem na qualidade de vida do paciente com LP'.

Chacon e colaboradores ${ }^{10}$ identificaram os custos de tratamento de LP estágios $3 \mathrm{e} 4 \mathrm{em}$ pacientes hospitalizados, sendo que o gasto médio calculado foi de $\mathrm{R} \$ 8.889,63$. Dealey, Posnett e Walker ${ }^{11}$ identificaram custos para tratamento de LP na Inglaterra e houve variação de $£ 1.064,00$ para estágio 1 até $£ 10.551,00$ para estágio 4 .

A introdução da tecnologia informática e o surgimento de gadgets sofisticados, como computadores, notebooks, palmtops, tablets, smartphones e telefones portáteis com suporte de internet trouxeram muitos benefícios para a assistência em saúde, permitindo aos profissionais o acesso, em qualquer lugar ou hora, a uma grande quantidade de informações sobre o paciente ou sua doença ${ }^{12}$. Existem recursos para orientação de profissionais de saúde sobre o tema, como sites específicos, aulas, textos, diretrizes. Dentre esses recursos, destaca-se o aplicativo ( $a p p$ ) para smartphone, que é um tipo de software que permite ao usuário realizar tarefas específicas. Existem para computadores e para celulares ou smartphones, chamados mobileapp $s^{13}$.

O smartphone é considerado uma ferramenta de fácil uso, que pode atingir uma grande parcela de enfermeiros e outros profissionais da equipe de saúde ${ }^{14}$. Os smartphones revolucionaram a forma como as pessoas se comunicam atualmente. Eles fornecem serviços de informações sem fio, incluindo navegação na internet, telas sensíveis com alta resolução, acesso de dados em alta velocidade via $\mathrm{Wi}-\mathrm{Fi}$ e banda larga, além de alta capacidade da câmera digita ${ }^{14}$.

As tecnologias móveis de comunicação e informação podem ajudar a implementar protocolos de cuidados com úlceras e a monitorização de doentes com alto risco, prevenindo ou melhorando essas condições ${ }^{15}$. A maneira de interagir na atualidade é intensamente influenciada pelas tecnologias de informação e comunicação, sendo de muita importância intensificar esses tipos de alternativas nos diversos cenários da formação em saúde, usando-as para fins específicos e com objetivos benéficos ${ }^{16}$.

Portanto, é necessário o desenvolvimento de recursos como aplicativos que contenham arquivos de referência para a correta identificação, estadiamento e prevenção de LP. $\mathrm{O}$ acesso ao conhecimento facilita o diagnóstico precoce, diminuindo o tempo e o custo do tratamento. Além disso, proporciona a implementação de medidas de prevenção, fundamental na redução de LP e suas complicações, bem como na garantia da segurança do paciente.
Assim, o objetivo deste estudo foi desenvolver um aplicativo que possa ser utilizado por profissionais de saúde, com orientações para identificação, estadiamento e prevenção de LP em adultos.

\section{MÉTODO}

Estudo metodológico descritivo de desenvolvimento de um aplicativo para smartphone para orientação sobre identificação, estadiamento e prevenção de LP em adultos. O estudo foi aprovado pelo Comitê de Ética em Pesquisa da Universidade Federal de São Paulo (UNIFESP), com CAAE 78020117.0.0000.5505, de 18 de outubro de 2017.

$\mathrm{O}$ trabalho iniciou com a busca de anterioridade nas plataformas Google $\odot$ e Apple $\odot$, pesquisando-se a existência de aplicativos na área de prevenção e tratamento de LP. As palavras-chave utilizadas na busca foram: Wounds, Pressure Ulcer, Pressure Sore, Pressure Injury, Decubitus Ulcer, Ferida, Úlcera por Pressão, Úlcera de Decúbito, Lesão por Pressão. Foram considerados para a pesquisa somente aplicativos que oferecessem conhecimento científico na área, sendo excluídos aqueles para entretenimento. Em dezembro/2016, nas plataformas Google(C) e Apple $\odot$, foram encontrados 76 aplicativos na área de feridas e LP. Desses, 84\% estavam na língua inglesa e $16 \%$ em outras línguas, como francês, italiano e espanhol. Apenas um app foi encontrado em português, mas o seu uso é exclusivo para a equipe de um hospital do município de São Paulo. Quanto ao custo, 57 deles eram gratuitos e 19 pagos.

A busca de anterioridade também foi realizada nas bases de dados PUBMED, Scielo e Lilacs e na plataforma de busca Google Scholar. Foi encontrado um artigo relacionado a aplicativos na área de LP, não sendo o objetivo a prevenção dessa lesão, e sim seu monitoramento ${ }^{15}$.

A partir dessa busca, o aplicativo foi desenvolvido em cinco etapas: levantamento bibliográfico, desenvolvimento de conteúdo, programação e design na plataforma de criação, avaliação do aplicativo e disponibilização na plataforma para download.

O conteúdo do aplicativo foi baseado em artigos recentes publicados sobre LP nas recomendações da National Pressure Injury Advisory Panel (NPIAP), de 2014 e 2016, no Protocolo de Segurança do Paciente da Agencia Nacional de Vigilância Sanitária (ANVISA), de 2013, e no livro de Prevenção e Tratamento de Lesão por Pressão17. 
De forma a ilustrar e auxiliar na correta identificação das lesões foi criado um item: Imagens de Estadiamento. Esse item contém imagens próprias da autora (fotografadas com autorização prévia dos pacientes), obtidas durante a prática clínica prévia e, também, imagens da NPIAP de uso autorizado. Além das imagens, foi utilizada a Escala de Braden validada para a língua portuguesa, também com uso autorizado. Essa escala é um instrumento desenvolvido em 1987 por Barbara Braden e Nancy Bergstrom, que auxilia na avaliação de risco de desenvolvimento de LP.

A programação do protótipo foi realizada em um framework disponível no site gratuito www. fábricadeaplicativos.com.br. Foram criadas sete abas: Informações do Aplicativo, Conceito e Riscos, Estadiamento, Imagens de Estadiamento, Escala de Braden, Prevenção e Referências.

A avaliação do aplicativo foi baseada no método Design Thinkink ${ }^{18}$, que é uma abordagem à resolução de problemas que se baseia em: empatia, colaboração e experimentação. Quando um grupo de pessoas analisa um problema em conjunto, leva a soluções novas sobre aquele problema. O Design Thinking gera solução de problemas de formas diferentes, criando novas opções aos diferentes aspectos, que vão além de melhorarem as alternativas existentes. No momento em que são geradas as alternativas, volta-se à experimentação, que é chamada de prototipação, a qual não é apenas construir e verificar se funciona, mas também um processo evolutivo ${ }^{18}$.

Após o protótipo pronto, foi enviado para avaliação de participantes da equipe de saúde, em uma amostra de conveniência de nove profissionais. A amostra de conveniência foi utilizada devido ao custo e tempo menores dispendidos nessa amostragem. A participação dos avaliadores foi realizada após concordância em participar do estudo e assinatura do Termo de Consentimento Livre e Esclarecido (TCLE). O processo de avaliação do app foi realizado em duas fases. $\mathrm{Na}$ primeira fase foi elaborado um questionário para profissionais de saúde da amostra. O questionário possuía perguntas elaboradas pela autora, relacionadas ao conteúdo e à forma do aplicativo, e as respostas poderiam ser respondidas como SIM ou NÃO e ao final havia uma opção para inclusão de comentários e sugestões. Os profissionais receberam o link para acesso ao aplicativo e responderam ao questionário enviado por e-mail, em que analisaram a estrutura, o conteúdo e a acessibilidade da linguagem. Após a avaliação desses usuários, foram feitas melhorias sugeridas até que o app estivesse com estrutura, conteúdo e linguagem adequados à prática do profissional. Após essa fase e a adequação das sugestões, iniciou-se a fase dois da avaliação do $a p p$. Dessa vez, três enfermeiros estomaterapeutas participaram. Essa categoria de profissionais foi escolhida, pois foi a única que sugeriu alterações na primeira fase da avaliação. Todos os três participantes enviaram o formulário concordantes com todos os itens. O aplicativo foi, então, considerado adequado na forma e conteúdo para disponibilização.

A partir do protótipo avaliado e atualizado, seguiu-se a programação do final do aplicativo e a disponibilização nas plataformas para download gratuito.

\section{RESULTADOS}

O aplicativo desenvolvido a partir da página "fábrica de aplicativos” foi denominado “Sem Pressão” (Fig. 1).

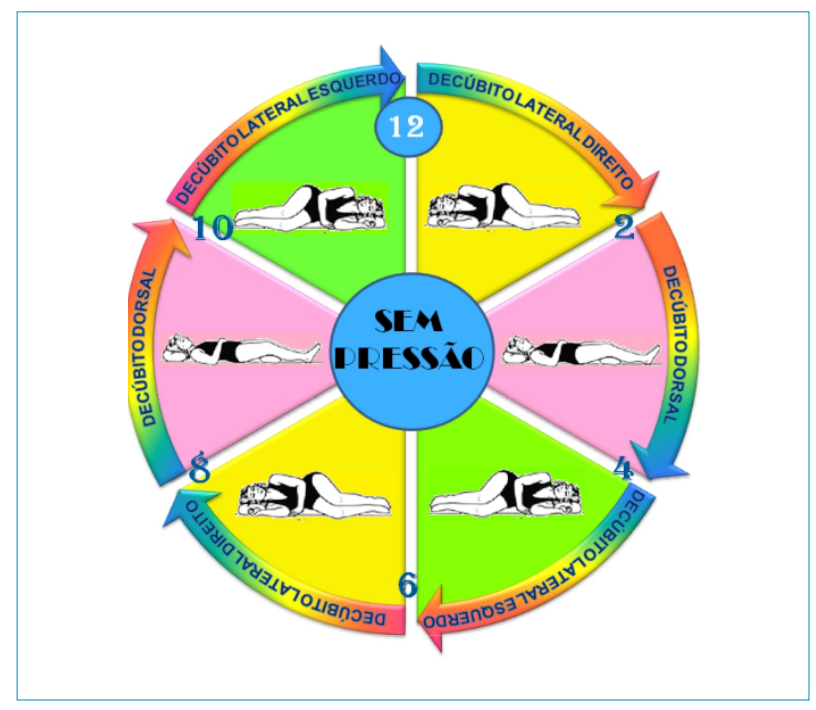

Figura 1. Tela inicial do aplicativo SEM PRESSÃO. São Paulo (SP), Brasil - 2017.

O nome do aplicativo surgiu da principal causa da lesão: a pressão. Sem a pressão, o risco de desenvolvimento dessa ferida é ausente. Além disso, a ocorrência desse tipo de ferida é utilizada como avaliação de qualidade da assistência ao paciente. Sendo assim, profissionais de saúde sentem-se pressionados a estabelecer programas preventivos. A criação do nome do aplicativo juntou esses dois elementos e foi denominado: “Sem Pressão”. 
Ao abrir o aplicativo, a tela inicial apresenta o layout desenvolvido. A seguir, automaticamente, segue a página com o menu inicial (Fig. 2).

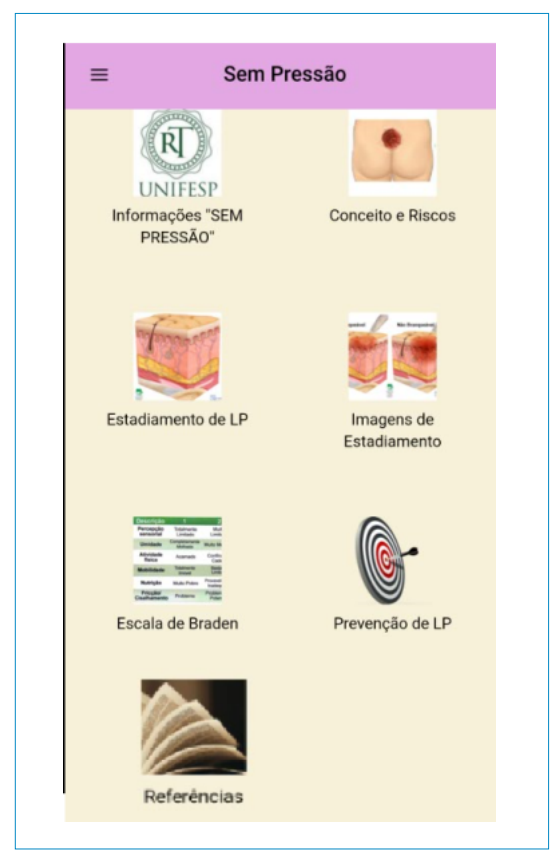

Figura 2. Tela de menu inicial do aplicativo SEM PRESSÃO. São Paulo (SP), Brasil - 2017.

O menu inicial (Fig. 2) contém as abas: Informações, Conceito e Riscos, Estadiamento, Imagens de Estadiamento, Escala de Braden, Prevenção de LP e Referências. Foi incluída uma aba, "Informações", para que o usuário conheça a origem do aplicativo, os autores e o curso de mestrado em que o aplicativo foi desenvolvido. A próxima aba, "Conceito e Riscos", foi subdividida em três itens: Conceito, Fatores de Risco e Regiões de Risco. O conteúdo contempla o conceito de LP segundo a NPIAP, os principais fatores de risco e também as regiões corporais de risco.

A Fig. 3 apresenta a aba "Estadiamento de LP". Foi incluída a classificação da NPIAP": LP estágios 1 a 4, LP Não Classificável, LP Tissular Profunda, LP por dispositivos e LP em membranas mucosas.

A tela seguinte (Fig. 4) apresenta uma sequência de imagens de LP. Cada classificação tem um ou mais exemplos em fotos para elucidar os conceitos. Ao clicar em uma foto, abre-se uma descrição do estágio e localização da lesão apresentada.

A escala de Braden foi incluída em uma aba específica. Essa aba foi ampliada em itens, em que foram descritas cada subescala. Ao clicar em um item ele se abre com a descrição. A seguir o aplicativo apresenta o item "Prevenção". Esse item foi dividido em 11 subitens (Fig. 5): Conceito, Inspeção e Cuidados com a Pele, Incontinência, Nutrição e Hidratação, Reposicionamento, Redistribuição da Pressão, Coberturas Profiláticas, LP no Paciente Sentado, LP Relacionada a Dispositivos, Prevenção na Sala Cirúrgica e Educação para Prevenção.

A última aba foi denominada "Referências". Foram colocadas as principais bibliografias utilizadas no desenvolvimento do conteúdo do aplicativo.

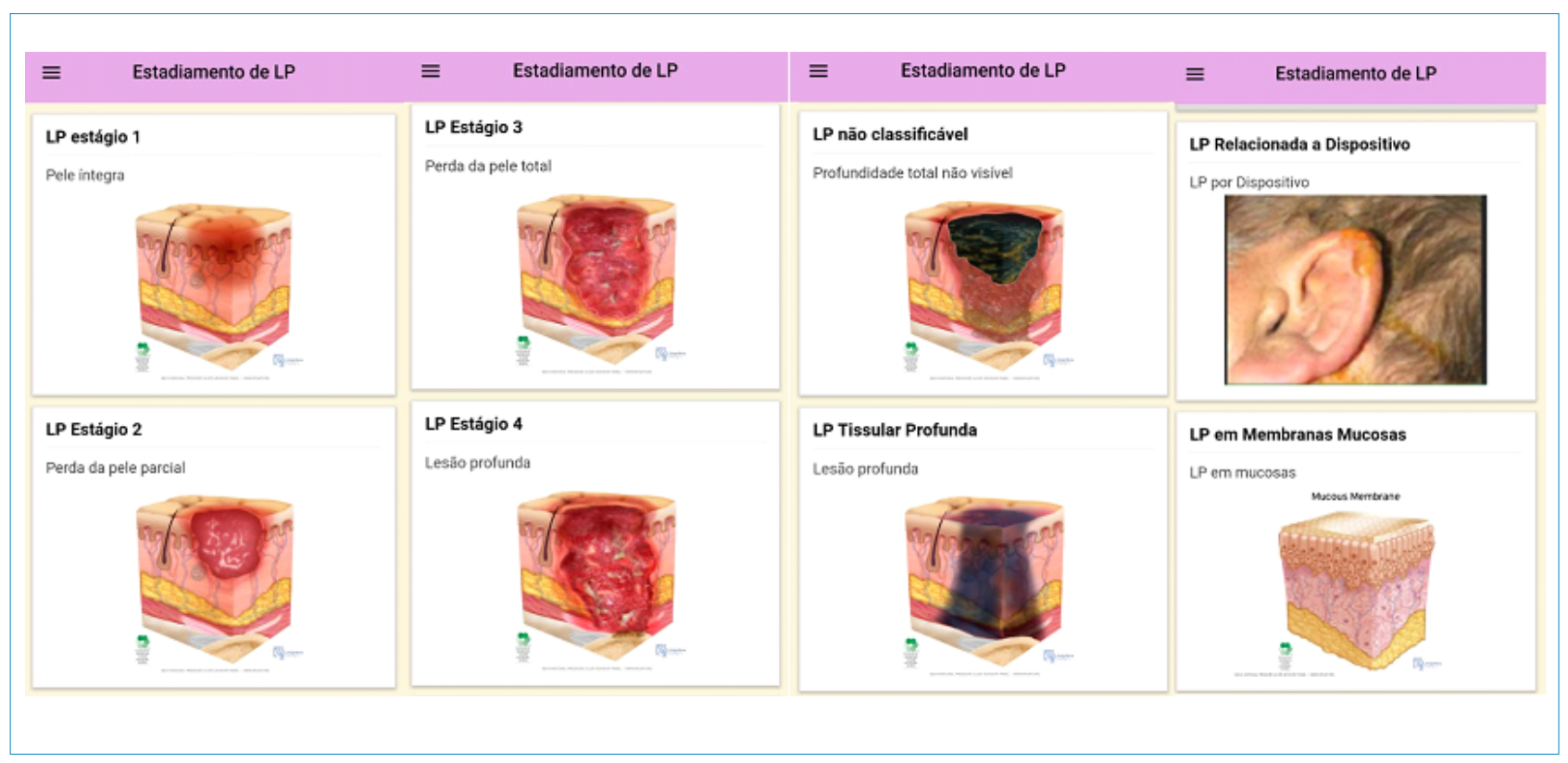

Figura 3. Estadiamento de lesões por pressão do aplicativo SEM PRESSÃO. São Paulo (SP), Brasil - 2017. 


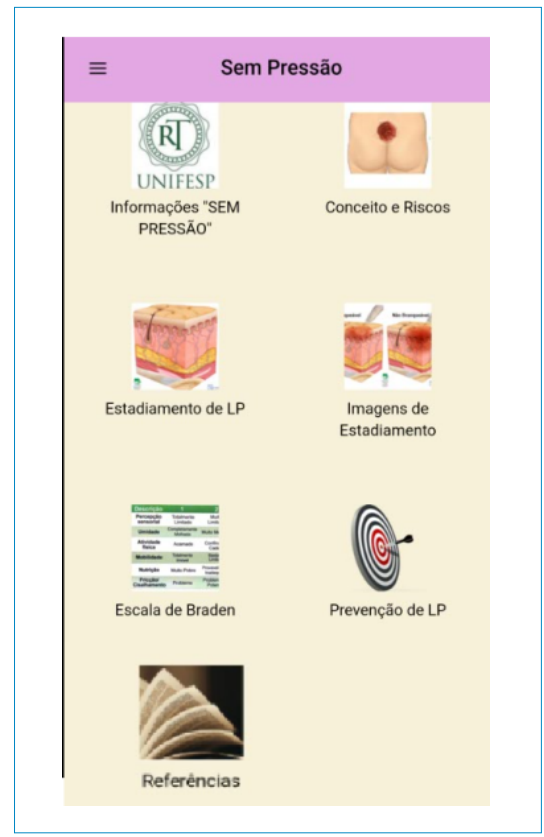

Figura 4. Imagens de estadiamento de lesão por pressão do aplicativo SEM PRESSÃO. São Paulo (SP), Brasil - 2017.

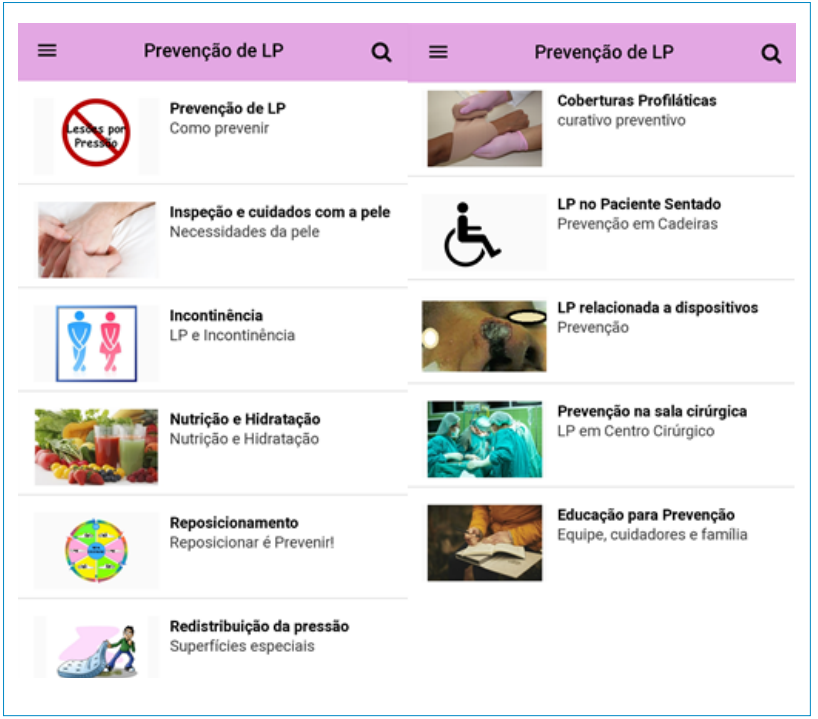

Figura 5. Prevenção e subitens do aplicativo SEM PRESSÃO. São Paulo (SP), Brasil - 2017.

\section{DISCUSSÃO}

As evidências sugerem que as LP podem ser prevenidas com a implementação de diretrizes de prevenção que visam a identificação de fatores de risco conhecidos associados ao desenvolvimento de $\mathrm{LP}^{19,20}$. Portanto, a identificação e a compreensão dos fatores de risco são necessárias para fornecer intervenções de prevenção adequadas e utilizar melhor os recursos na prática ${ }^{10}$.
A abordagem do tema no aplicativo "Sem Pressão" foi voltada para profissionais de saúde, para identificação, estadiamento e prevenção em pacientes adultos. A escolha do público adulto se deu pela grande quantidade e variedade de publicações disponíveis sobre prevenção de LP. A sequência de conteúdo do aplicativo foi seguida conforme aparece nas diretrizes conhecidas de prevenção de LP como da NPIAP. Os conceitos apresentados são os mais recentes ${ }^{1,17,20}$.

O design do aplicativo "Sem Pressão" foi disponibilizado pela plataforma de criação: www.fabricadeaplicativos.com. br. A plataforma é simples de utilizar, com modelos prontos e customizáveis. Foram escolhidas cores de fundo suaves, que não interferissem no aspecto educativo do aplicativo. A plataforma de criação foi escolhida pela facilidade, acessibilidade e baixo custo. Os pontos positivos do uso desse método foram a rapidez de desenvolvimento e atualização, além da praticidade em divulgar o link para teste e mesmo para download. No entanto, há limites, como a rigidez da plataforma que não permite interações que um aplicativo educativo necessita, por exemplo, possibilitar a inserção de dados e o cálculo automático dos escores na escala de Braden. As ilustrações de estadiamento foram autorizadas para utilização no app pela NPIAP e as demais foram encontradas em sites de imagens gratuitas como o pixabay.com.

Garantir a eficácia e a segurança dos aplicativos para uso na saúde é fundamental dada a variabilidade de aplicativos atualmente disponíveis. Além de demonstrar a precisão, eficácia ou segurança de um determinado $a p p$, a avaliação rigorosa dessas ferramentas clínicas também pode revelar resultados não previstos durante o desenvolvimento ${ }^{21,14}$. A avaliação do conteúdo do "Sem Pressão" foi baseada na metodologia Design Thinking e realizada por profissionais da equipe de saúde, experientes na dispensação do cuidado relativo à LP. Essa avaliação teve por objetivo dar credibilidade ao $a p p$, bem como garantir que as informações presentes nele fossem coerentes com as diretrizes internacionais e com a prática clínica. A amostra de conveniência utilizada contemplou um profissional fisioterapeuta que atua na mobilização precoce e prevenção de LP em UTI, uma psicóloga hospitalar, três enfermeiros estomaterapeutas experientes na área de LP e quatro enfermeiros generalistas atuantes na área hospitalar com pacientes acamados em risco de LP.

No retorno recebido das avaliações dos profissionais, um ponto relevante foi a inclusão de prevenção de LP em 
centro cirúrgico. Tallier e colaboradores ${ }^{22}$, em estudo sobre prevenção de LP nesse setor, identificaram que enfermeiros do centro cirúrgico têm um déficit de conhecimento sobre avaliação e prevenção de LP e, ainda, que esses profissionais não se envolvem em atividades de educação continuada a respeito de LP. Assim, um aplicativo que possui um item específico de prevenção em centro cirúrgico vem apoiar na tomada de decisão sobre prevenção em pacientes operatórios, com acesso rápido e pontual, propiciando aumento de conhecimento e ações preventivas de fato.

$\mathrm{O}$ acesso oportuno a informações clínicas é um fator chave para garantir a prática assistencial eficaz e segura. A tecnologia dos aplicativos em smartphones tem potencial de melhorar a acessibilidade a dados e recursos como as evidências clínicas e científicas. Sendo assim, o uso de smartphones por profissionais de saúde pode ajudar a melhorar os padrões de atendimento ${ }^{23}$. Esse tipo de tecnologia ganha espaço no cotidiano das pessoas, influenciando diretamente políticas públicas e levando a sociedade a agregar novos valores socioculturais e democráticos, exercendo fundamentalmente a função de recurso educacional e informativo ${ }^{16}$. O download de aplicativos pode ser feito de qualquer lugar do mundo, desde que se tenha acesso à internet ${ }^{24}$. Isso possibilita que um país continental como o Brasil alcance essa ferramenta de conhecimento nos locais mais distantes de grandes centros. Além disso, os profissionais carregam seus equipamentos consigo, assim, o acesso à informação acontecerá em qualquer lugar, sem a dependência de um consultório ou computador fixo.

A provisão de informações atualizadas e precisas é essencial para apoiar os profissionais no momento em que estão gerenciando o atendimento ao paciente ${ }^{15}$. Um aplicativo móvel que permite o acesso oportuno, sem gasto de internet apoia a aprendizagem e a prática de profissionais recémformados ${ }^{25}$. Agu e colaboradores ${ }^{24}$ citam que smartphones ficam junto a seus usuários em $90 \%$ do tempo. Essa vantagem apresenta-se ainda maior para os enfermeiros de cuidado domiciliar, pois podem ter acesso à informação a qualquer momento, aumentando a eficiência de suas ações diagnósticas e preventivas ${ }^{15}$.

A prevenção da ocorrência de LP traz benefícios, como redução de custos, melhor cicatrização (em caso de existência de LP), diminuição da necessidade de intervenção cirúrgica como desbridamento ou enxertia, redução do tempo de internação hospitalar ou domiciliar, entre outros ${ }^{15}$. Assim, o "Sem Pressão" pode auxiliar as instituições na redução de custos associados à assistência, além da redução de danos iatrogênicos da hospitalização.

A individualização do cuidado através da sistematização da assistência de enfermagem e o uso de recomendações para as práticas clínicas baseadas em evidências podem melhorar a qualidade do cuidado e proporcionar benefícios ao paciente23. A criação de um aplicativo em português e gratuito para download certamente é uma ferramenta que disseminará conhecimento aos profissionais, principalmente aos não especialistas, que, assim, poderão tomar iniciativas no sentido de prevenir LP.

\section{CONCLUSÃO}

Foi desenvolvido aplicativo gratuito e em português, com orientações sobre identificação, estadiamento e prevenção de LP em adultos, denominado "Sem Pressão" e disponibilizado para download gratuito na Google Play Store ${ }^{\mathrm{TM}}$.

\section{CONTRIBUIÇÃO DOS AUTORES}

Conceitualização: Campos RS, Blanes L; Metodologia: Campos RS, Blanes L, Ferreira LM; Redação - Primeira versão: Campos RS, Blanes L; Redação - Revisão \& Edição: Campos RS, Blanes L, Nicodemo D, Ferreira LM; Aquisição de Financiamento: Campos RS; Recursos: Campos RS; Supervisão: Blanes L, Nicodemo D, Ferreira LM.

\section{REFERÊNCIAS}

1. European Pressure Ulcer Advisory Panel, National Pressure Injury Advisory Panel and Pan Pacific Pressure Injury Alliance. Prevention and treatment of pressure ulcers/injuries: clinical practice guideline. The international guideline. Emily Haesler (Ed.). EPUAP/NPIAP/PPPIA: 2019.
2. Caliri MHL, Santos VLCG, Mandelbaum MHS, Costa IG. Classificação das lesões por pressão - Consenso NPUAP 2016 - adaptada culturalmente para o Brasil. Publicação oficial da Associação Brasileira de Estomaterapia (SOBEST) e da Associação Brasileira de Enfermagem em Dermatologia 
(SOBENDE). 2016. [Acesso em 01 dez 2016]. Disponível em: http://sobest.org.br/textod/35

3. Galetto SGS, Nascimento ERP, Hermida PMV, Malfussi LBH. Lesões por Pressão Relacionadas a Dispositivos Médicos: revisão integrativa da literatura. Rev. Bras. Enferm 2019;72(2):528-36. https://doi.org/10.1590/0034-7167-2018-0530

4. Black JM, Kalowes P. Medical device-related pressure ulcers. Chronic Wound Care Manag Res 2016;3:91-9. https://doi. org/10.2147/CWCMR.S82370

5. Barbosa JM, Salomé GM. Ocorrência de lesão por pressão em pacientes internados em um hospital-escola. ESTIMA, Braz J Enterostomal Ther 2018;16:e2718. https://doi. org/10.30886/estima.v16.523_PT

6. Constantin AG, Moreira APP, Oliveira JLC, Hofstätter LM, Fernandes LM. Incidência de lesão por pressão em unidade de terapia intensiva para adultos. ESTIMA, Braz J Enterostomal Ther 2018;16:e1118. https://doi. org/10.30886/estima.v16.454_PT

7. Pittman J, Beeson T, Kitterman J, Lancaster S, Shelly A. Medical device-related hospital-acquired pressure ulcers. Development of an evidence-based position statement. J Wound Ostomy Continence Nurs 2015;42(2):151-4. https:// doi.org/10.1097/WON.0000000000000113

8. Mazzo A, Miranda FBG, Meska MHG, Bianchini A, Bernardes RM, Pereira Junior GA. Teaching of pressure injury prevention and treatment using simulation. Esc. Anna Nery 2018;22(1):e20170182. https://doi.org/10.1590/2177-9465ean-2017-0182

9. Galhardo VAC, Magalhães MG, Blanes L, Juliano Y, Ferreira LM. Health-related quality of life and depression in older patients with pressure ulcers. Wounds 2010;22(1):20-6. Disponível em: https://www.woundsresearch.com/content/ health-related-quality-life-and-depression-older-patientswith-pressure-ulcers

10. Chacon JMF, Blanes L, Borba LG, Rocha LRM, Ferreira LM. Direct variable cost of the topical treatment of stages III and IV pressure injuries incurred in a public university hospital. J Tissue Viability 2017;26(2):108-12. https://doi.org/10.1016/j. jtv.2016.12.003

11. Dealey C, Posnett J, Walker A. The cost of pressure ulcers in the United Kingdom. J Wound Care 2012;21(6):261-6. https://doi.org/10.12968/jowc.2012.21.6.261

12. Vêscovi SJB, Primo CC, Sant'Anna HC, Bringuete MEO, Rohr RV, Prado TN et al. Mo-bile application for evaluation of feet in people with diabetes mellitus. Acta Paul Enferm 2017;30(6):60713. https://doi.org/10.1590/1982-0194201700087

13. Rouse M. mHealth (mobile health) in: TechTarget [internet]. [Acesso em 30 out 2016]. Disponível em: http:// searchhealthit.techtarget.com/definition/mHealth

14. Sving E, Fredriksson L, Gunningberg L, Mamhidir A-G. Getting evidence-based pressure ulcer prevention into practice: a process evaluation of a multifaceted intervention in a hospital setting. J Clin Nurs 2017;26(19-20):3200-11. https://doi.org/10.1111/jocn.13668
15. Rodrigues JJPC, Pedro LMCC, Vardasca T, Torre-Díez I, Martins HMG. Mobile health platform for pressure ulcer monitoring with electronic health record integration. Health Informatics J 2013;19(4):300-11. https://doi. org/10.1177/1460458212474909

16. Pereira FGF; Frota NM; Silva DV; Sousa LMO, Almeida JC; Cysne Filho FMS. Avaliação de aplicativo digital para o ensino de sinais vitais. Rev Min Enferm 2017;21:e1034. https://doi. org/10.5935/1415-2762.20170044

17. Blanes L, Ferreira LM. Prevenção e tratamento de úlcera por pressão. $1^{\text {a }}$ ed. Rio de Janeiro: Atheneu; 2013.

18. Ferreira FK, Song EH, Gomes H, Garcia EB, Ferreira LM. New mindset in scientific method in the health field: design thinking. Clinics (São Paulo) 2015;70(12):770-2. https://doi. org/10.6061/clinics/2015(12)01

19. Coyer F, Tayyib N. Risk factors for pressure injury development in critically ill patients in the intensive care unit: a systematic review protocol. Syst Rev 2017;6:58. https://doi. org/10.1186/s13643-017-0451-5

20. Association of perioperative Registered Nurses [homepage]. Position statement on perioperative pressure ulcer prevention in the care of the surgical patient. AORN Journal 2016;104(5). Disponível em: https://aornjournal. onlinelibrary.wiley.com/doi/10.1016/j.aorn.2016.08.011

21. Pokorná A, Jarkovský J, Mužík J, Vasmanská S, Saibertová S, Krejčiříková P. A new online software tool for pressure ulcer monitoring as an educational instrument for unified nursing assessment in clinical settings. Mefanet Journal 2016; [citado em mar 2020]; 4(1):26-32. Disponível em: https:// www.researchgate.net/publication/305810458_A_new_ online_software_tool_for_pressure_ulcer_monitoring_as_an_ educational_instrument_for_unified_nursing_assessment_ in_clinical_settings

22. Tallier PC, Reineke PR, Asadoorian K, Choonoo JG, Campo $\mathrm{M}$, Wallen CM. Perioperative registered nurses knowledge, attitudes, behaviors, and barriers regarding pressure ulcer prevention in perioperative patients. Appl Nurs Res 2017;36:106-10. https://doi.org/10.1016/j.apnr.2017.06.009

23. Mercês JMR, Redeiro MMP. A importância dos dispositivos móveis como estratégia para a formação e desenvolvimento de profissionais de saúde. UERJ 2016; [citado em fev 2020]; Disponível em: http://www.abed.org.br/congresso2016/ trabalhos/306.pdf

24. Agu E, Pedersen P, Strong D, Tulu B, He Q, Wang L et al. The smartphone as a medical device: assessing enablers, benefits and challenges. IEEE International Workshop of Internet-of-Things Networking and Control (IoT-NC) 2013:76-80. https://doi.org/10.1109/loT-NC.2013.6694053

25. Bullock A, Dimond R, Webb K, Lovatt J, Hardyman W, Stacey M. How a mobile app supports the learning and practice of newly qualified doctors in the UK: an intervention study. BMC Med Educ 2015;15:71. https://doi.org/10.1186/s12909-0150356-8 